\title{
Hydrazine Complexes of Lanthanides with 3-Acetoxy- and 4-Acetoxybenzoic Acids: Spectroscopic, Thermal, and XRD Studies
}

\author{
E. Helen Pricilla Bai ${ }^{1}$ and S. Vairam ${ }^{2}$ \\ ${ }^{1}$ Department of Chemistry, Park College of Engineering and Technology, Kaniyur, Coimbatore 641659, India \\ ${ }^{2}$ Department of Chemistry, Government College of Technology, Coimbatore 641013, India
}

Correspondence should be addressed to S. Vairam; vamshen@yahoo.com

Received 29 January 2012; Revised 20 May 2012; Accepted 29 May 2012

Academic Editor: Satoru Tsushima

Copyright (C) 2013 E. Helen Pricilla Bai and S. Vairam. This is an open access article distributed under the Creative Commons Attribution License, which permits unrestricted use, distribution, and reproduction in any medium, provided the original work is properly cited.

\begin{abstract}
New bis-hydrazine lanthanide complexes with 3-acetoxybenzoic acid (3-abH) of formula, $\left[\mathrm{Ln}(3-\mathrm{ab})_{3}\left(\mathrm{~N}_{2} \mathrm{H}_{4}\right)_{2}\right] \cdot x \mathrm{H}_{2} \mathrm{O}$ where $\mathrm{Ln}=$ $\mathrm{La}, \mathrm{Ce}, \mathrm{Pr}$ and $\mathrm{Gd}$ and $x=0$; $\mathrm{Ln}=\mathrm{Nd}$ and $\mathrm{Sm}$ and $x=1$, and monohydrazine complexes of some trivalent lanthanides with 4-acetoxybenzoic acid (4-abH) of formula, $\left[\mathrm{Ln}(4-\mathrm{ab})_{3}\left(\mathrm{~N}_{2} \mathrm{H}_{4}\right)\right] \cdot \mathrm{H}_{2} \mathrm{O}$ where $\mathrm{Ln}=\mathrm{La}, \mathrm{Ce}, \mathrm{Pr}, \mathrm{Nd}$, Sm and Gd have been prepared in ethanolic medium and characterized by spectroscopic techniques (IR and UV reflectance), microelemental analysis, thermoanalytical technique, powder XRD, SEM-EDS studies, and magnetic susceptibility measurements. The IR spectra of both series show bidental coordination of carboxylate ion with the metal by displaying, $v_{\mathrm{C}=\mathrm{O}(\text { asym) }}$ in the range of $1587-1602 \mathrm{~cm}^{-1}$ and the $v_{\mathrm{C}=\mathrm{O}(\mathrm{sym})}$ in the range of $1433-1410 \mathrm{~cm}^{-1}$, with a separation of around $200 \mathrm{~cm}^{-1}$. The ester $\mathrm{C}=\mathrm{O}$ remains unaltered indicating the noninvolvement in coordination. All the complexes show $v_{\mathrm{N}-\mathrm{N}}$ absorption in the range of $929-962 \mathrm{~cm}^{-1}$ indicating the presence of hydrazine in a bridged bidentate fashion. The thermal data reveals that the hydrated compounds show endothermic dehydration followed by exothermic decomposition to leave their metal oxide as end products, and the anhydrous compounds undergo exothermic decomposition to form the respective metal oxide residues.
\end{abstract}

\section{Introduction}

Salicylic acid plays vital role in coordination chemistry due to its capacity for chelation. One of its acetyl derivatives, aspirin, has also been used in the preparation of complexes due to its therapeutic applications [1-3]. It has been used as an axial ligand for the preparation of tin porphyrin complexes [4]. The other two isomers of aspirin, namely, 3-acetoxybenzoic acid $(3-\mathrm{abH})$ and 4 -acetoxybenzoic acid $(4-\mathrm{abH})$ have not been used for synthesis of complexes yet. In our laboratory, we have been synthesising metal carboxylate complexes using hydrazine as coligand [5-7]. When hydrazine is used as a coligand, it generally leads to the formation of polymeric complexes owing to its action as bridging ligand [8-11]. With the idea of preparing new hydrazine complexes, we selected the 3 and 4-acetoxybenzoic acids. In this paper, we have presented the preparation and the characterization of 3-and 4-acetoxybenzoic acid complexes of some lighter lanthanides with hydrazine as coligand by IR, uv-visible spectroscopic studies, thermoanalytical, XRD, SEM-EDX, and magnetic susceptibility measurement studies.

\section{Experimental}

The solvents were distilled prior to use, and double distilled water was used for the preparation and chemical analyses. The chemicals used were of AR grade. In all the reactions, 99.99\% pure hydrazine hydrate was used as received.

2.1. Preparation of $\left[\mathrm{Ln}(3-a b)_{3}\left(\mathrm{~N}_{2} \mathrm{H}_{4}\right)_{2}\right] \cdot \mathrm{xH}_{2} \mathrm{O}$ Where $\mathrm{Ln}=\mathrm{La}$, Ce, Pr, and Gd and $x=0 ; L n=N d$ and Sm and $x=$ 1. Lanthanum oxide $(0.325 \mathrm{~g}, 1 \mathrm{mmol})$ was dissolved in a minimum quantity of $1: 1 \mathrm{HNO}_{3}$, evaporated to eliminate excess of acid, and dissolved in $20 \mathrm{~mL}$ of ethanol. This 


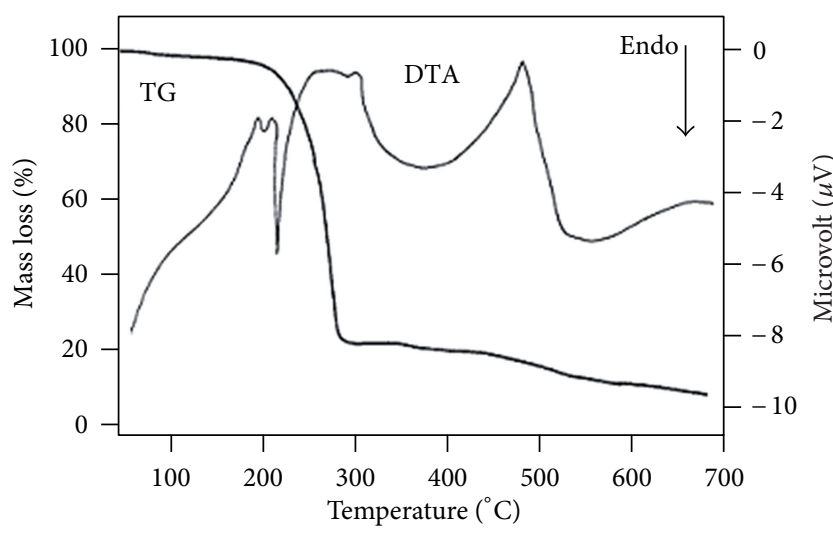

Figure 1: TG-DTA of $\left[\operatorname{Pr}(3-\mathrm{ab})_{3}\left(\mathrm{~N}_{2} \mathrm{H}_{4}\right)_{2}\right]$.

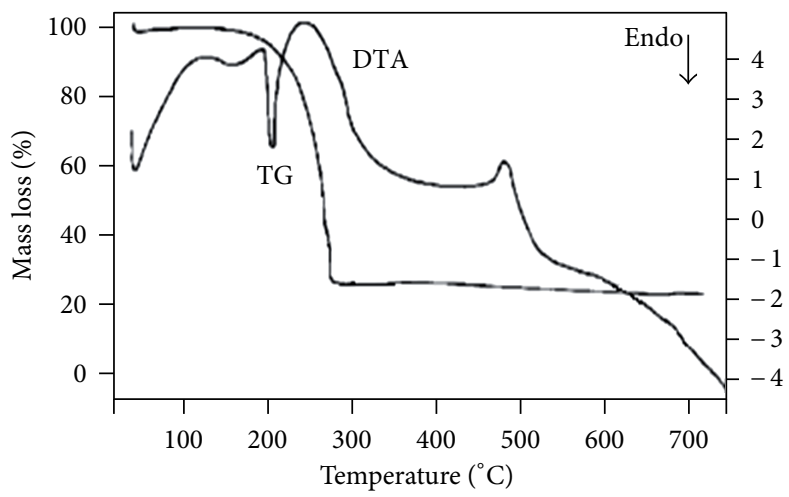

Figure 2: TG-DTA of $\left[\mathrm{Sm}(3-\mathrm{ab})_{3}\left(\mathrm{~N}_{2} \mathrm{H}_{4}\right)_{2}\right] \cdot \mathrm{H}_{2} \mathrm{O}$.

was added slowly to a freshly prepared ethanolic solution $(40 \mathrm{~mL})$ of the ligand containing $3-\mathrm{abH}(1.081 \mathrm{~g}, 6 \mathrm{mmol})$ and hydrazine hydrate $(0.40 \mathrm{~g}, 8 \mathrm{mmol})$, stirring the reaction mixture at $\mathrm{pH} 3$. Then the reaction mixture was kept over hot water bath for $1 \mathrm{~h} 30 \mathrm{~min}$. A dull white, crystalline product obtained was washed with alcohol, ether, and air dried. A similar procedure was adopted for obtaining the other lanthanides with the molar ratio Metal: Acid : Base $=1: 6: 8$.

2.2. Preparation of $\left[\mathrm{Ln}(4-a b)_{3}\left(\mathrm{~N}_{2} \mathrm{H}_{4}\right)\right] \cdot \mathrm{H}_{2} \mathrm{O}$ Where $\mathrm{Ln}=\mathrm{La}$, $\mathrm{Ce}, \mathrm{Pr}, \mathrm{Nd}, \mathrm{Sm}$ and $\mathrm{Gd}$. These complexes were prepared using $4-\mathrm{abH}$, hydrazine hydrate and their respective lanthanum nitrates in alcohol medium at $\mathrm{pH} 3.5$ by adopting similar procedure as mentioned above. The products obtained were washed with alcohol, ether and dried in air. All these complexes were prepared by using the molar ratio Metal : Acid : Base $=1: 6: 8$.

2.3. Physico chemical Methods. The hydrazine content in all complexes was determined volumetrically using $0.025 \mathrm{M}$ potassium iodate solution under Andrews' conditions [12]. The metal content was determined by EDTA complexometric titration [12] after decomposing a known weight of the sample with $1: 1 \mathrm{HNO}_{3}$. Magnetic measurements were carried out by the Gouy method using $\mathrm{Hg}\left[\mathrm{Co}(\mathrm{NCS})_{4}\right]$ as calibrant. The electronic spectra for solid-state complexes

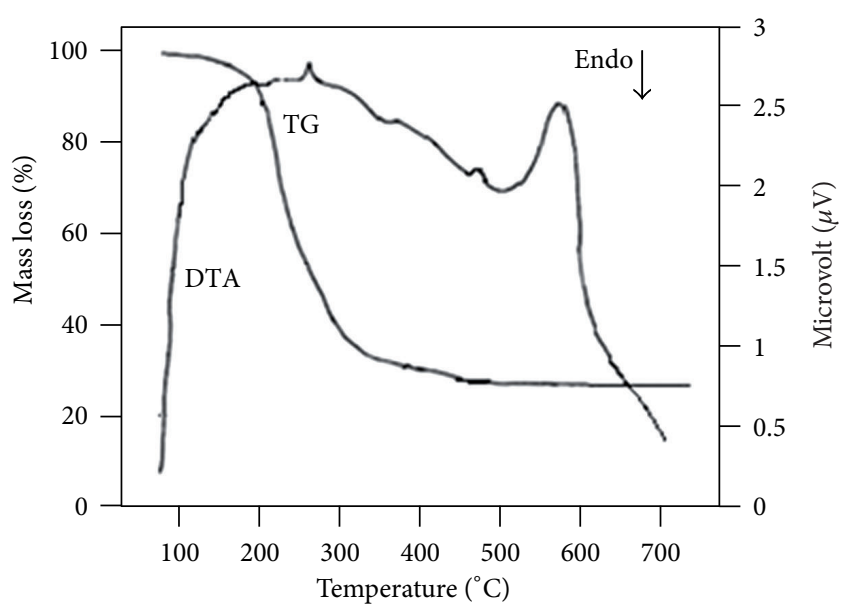

Figure 3: TG-DTA of $\left[\operatorname{Pr}(4-\mathrm{ab})_{3}\left(\mathrm{~N}_{2} \mathrm{H}_{4}\right)\right] \cdot \mathrm{H}_{2} \mathrm{O}$.

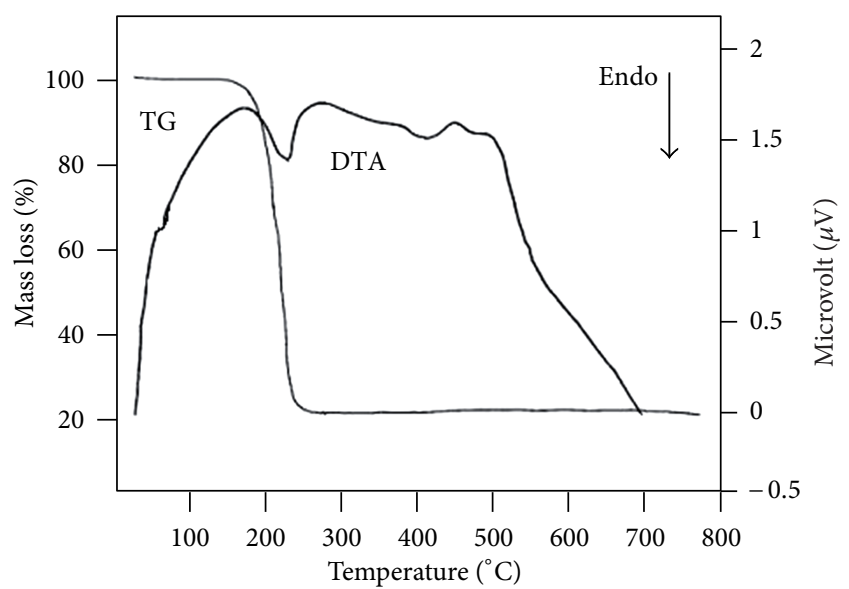

Figure 4: TG-DTA of $\left[\mathrm{Gd}(4-\mathrm{ab})_{3}\left(\mathrm{~N}_{2} \mathrm{H}_{4}\right)\right] \cdot \mathrm{H}_{2} \mathrm{O}$.

were obtained using a Varian, Cary 5000 recording spectrophotometer. Infrared spectra were recorded using KBR disc $\left(4000-400 \mathrm{~cm}^{-1}\right)$ on a Shimadzu FTIR-8201 (PC) S spectrophotometer. The simultaneous TG-DTA studies were done on a Perkin Elmer Diamond TG/DTA analyzer, and the curves were obtained in static air using 5-10 mg of the samples at the heating rate of $10^{\circ} \mathrm{C} / \mathrm{min}$. The XRD patterns were recorded on a Bruker AXS D8 advance diffractometer with an X-ray source $\mathrm{Cu}$, wavelength $1.5406 \AA$ using a $\mathrm{Si}(\mathrm{Li})$ PSD detector. The elemental analysis was carried out using a CHNS Elementar Vario EL III Elemental Analyzer. The SEM with EDX analysis was obtained using JEOL model JSM-6390 LV and JEOL model JED-2300 instrument.

\section{Results and Discussion}

All the complexes obtained were polycrystalline powders that are stable in air and insensitive to light. Lanthanum complex is sparingly soluble in water but other complexes were insoluble. They were also insoluble in organic solvents such as ethanol, ether, and benzene, but soluble in DMSO. 


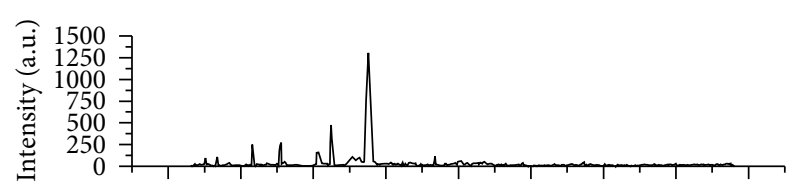

(f)

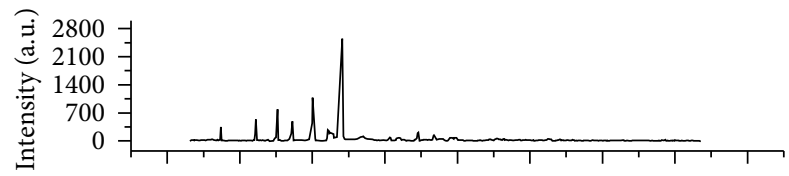

(e)

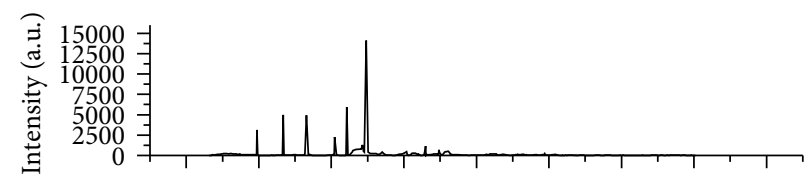

(d)

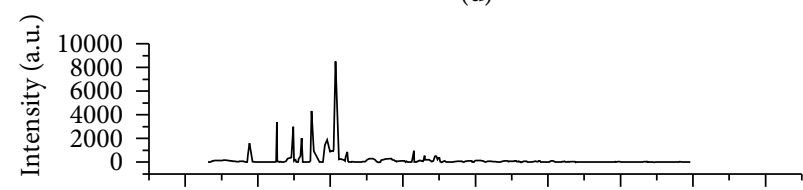

(c)

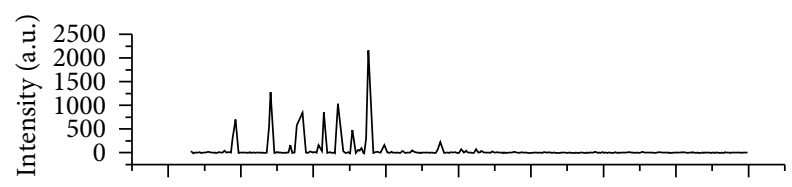

(b)

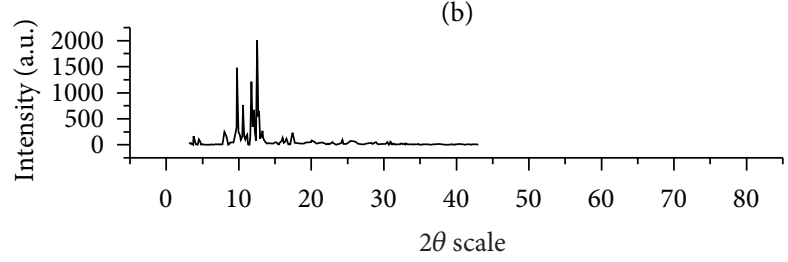

(a)

FIGURE 5: XRD patterns of lanthanide complexes of 3-abH. (a) [La(3-ab) $\left.{ }_{3}\left(\mathrm{~N}_{2} \mathrm{H}_{4}\right)_{2}\right]$. (b) [Ce(3-ab) $\left.)_{3}\left(\mathrm{~N}_{2} \mathrm{H}_{4}\right)_{2}\right]$. (c) $\left[\mathrm{Pr}(3-\mathrm{ab})_{3}\left(\mathrm{~N}_{2} \mathrm{H}_{4}\right)_{2}\right]$. (d) $\left[\mathrm{Nd}(3-\mathrm{ab})_{3}\left(\mathrm{~N}_{2} \mathrm{H}_{4}\right)_{2}\right] \cdot \mathrm{H}_{2} \mathrm{O}$. (e) $\left[\mathrm{Sm}(3-\mathrm{ab})_{3}\left(\mathrm{~N}_{2} \mathrm{H}_{4}\right)_{2}\right] \cdot \mathrm{H}_{2} \mathrm{O}$. (f) $\left[\mathrm{Gd}(3-\mathrm{ab})_{3}\left(\mathrm{~N}_{2} \mathrm{H}_{4}\right)_{2}\right]$.

The analytical data of the complexes is given in Table 1 and the values were consistent with the proposed formulae for them.

\subsection{Electronic Spectra and Magnetic Susceptibility Measure-} ments. The compounds were insoluble in water and organic solvents, and hence their electronic spectra were recorded for solid samples. The electronic spectral data and the assignments were summarized in Table 2 . The levels assigned were ${ }^{3} \mathrm{~F}_{4} \rightarrow{ }^{2} \mathrm{P}_{2}$ and ${ }^{4} \mathrm{I}_{15 / 2} \rightarrow{ }^{2} \mathrm{~F}_{3 / 2},{ }^{2} \mathrm{D}_{3 / 2}$ for $\mathrm{Nd}$ and Pr complexes of 4 -abH. Similarly the levels assigned for complexes of $\mathrm{Nd}$ and $\mathrm{Pr}$ of 3 -abH were ${ }^{2} \mathrm{H}_{11 / 2} \rightarrow{ }^{4} \mathrm{~S}_{3 / 2}$ and ${ }^{2} \mathrm{H}_{11 / 2} \rightarrow{ }^{3} \mathrm{~F}_{4}$, respectively [13, 14]. The effective magnetic moment values, $3.43,3.48 \mathrm{BM}$ corresponding to Pr and 3.51, $3.56 \mathrm{BM}$ for $\mathrm{Nd}$ complexes of $3-\mathrm{abH}$ and $4-\mathrm{abH}$, respectively, are in good agreement with the values reported [15].

3.2. IR Spectra of Complexes. The IR data of the complexes and that of the respective acids are given in Table 3. The spectrum of $3-\mathrm{abH}$ acid shows absorptions at $1091 \mathrm{~cm}^{-1}$, $1207 \mathrm{~cm}^{-1}, 1276 \mathrm{~cm}^{-1}$ due to $v_{\mathrm{C}-\mathrm{O} \text { ester }}, 1761 \mathrm{~cm}^{-1}$ due to $v_{\mathrm{C}=\mathrm{O} \text { ester, }}$, and $1676 \mathrm{~cm}^{-1}$ due to $v_{\mathrm{C}=\mathrm{O} \text { acid }}$.

Spectra of the complexes show broad spectrum $1246-1315 \mathrm{~cm}^{-1}$ and centered at $1315 \mathrm{~cm}^{-1}$ (La), $1277 \mathrm{~cm}^{-1}$ (Ce), $1300 \mathrm{~cm}^{-1}(\mathrm{Pr}), 1303 \mathrm{~cm}^{-1}(\mathrm{Nd}), 1300 \mathrm{~cm}^{-1}(\mathrm{Sm})$, and $1300 \mathrm{~cm}^{-1}(\mathrm{Gd})$ due to $v_{\mathrm{C}-\mathrm{O} \text { ester }}$. A medium sharp band observed at $1091 \mathrm{~cm}^{-1}$ for the acid is found at $1112 \mathrm{~cm}^{-1}$ for the complexes. These absorptions are found to shift in the same direction for all the complexes due to coordination [16].

Further, $v_{\mathrm{C}=\mathrm{O} \text { (asym) }}$ and $v_{\mathrm{C}=\mathrm{O} \text { (sym) }}$ are observed in the range of $1587-1602$ and $1433-1430 \mathrm{~cm}^{-1}$. The difference being less than $200 \mathrm{~cm}^{-1}$ may be because of their bidental coordination. The similar type of bidental coordination is found to occur in $\mathrm{Cu}$ aspirinate complexes [16-18]. The complexes show two sharp bands in the range of 1001-1005 and $929-962 \mathrm{~cm}^{-1}$ raising ambiguity about the assignment of coordination mode of hydrazine [19]. Since lanthanides 


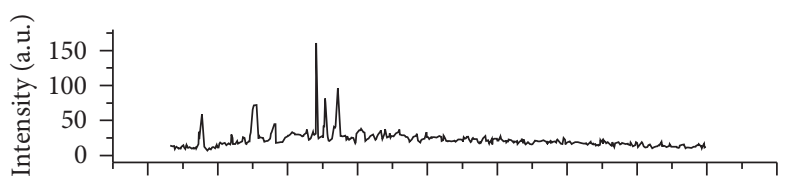

(f)

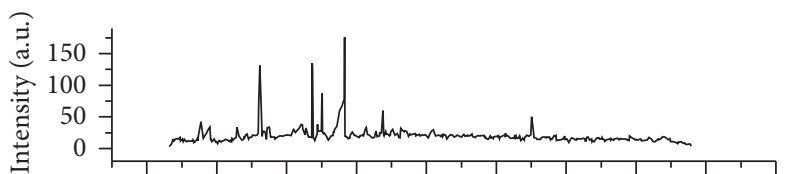

(e)

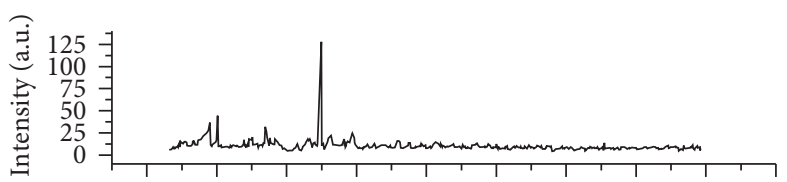

(d)

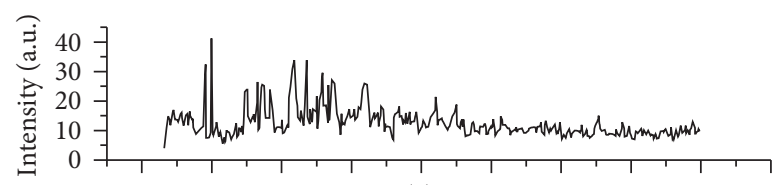

(c)

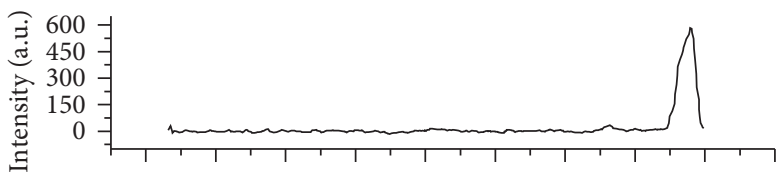

(b)

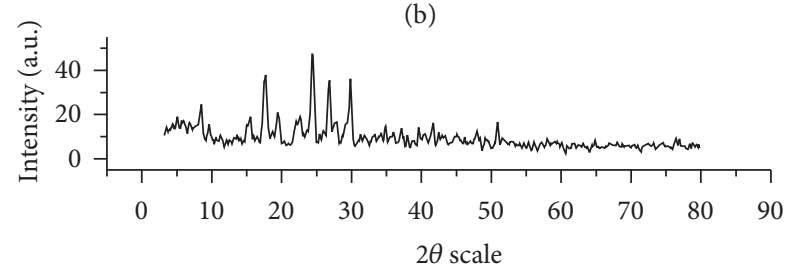

(a)

FIGURE 6: XRD patterns of lanthanide complexes of 4-abH. (a) [ $\left.\mathrm{La}(4-\mathrm{ab})_{3}\left(\mathrm{~N}_{2} \mathrm{H}_{4}\right)\right] \cdot \mathrm{H}_{2} \mathrm{O}$. (b) [Ce $\left.(4-\mathrm{ab})_{3}\left(\mathrm{~N}_{2} \mathrm{H}_{4}\right)\right] \cdot \mathrm{H}_{2} \mathrm{O}$. (c) [Pr(4$\left.\mathrm{ab})_{3}\left(\mathrm{~N}_{2} \mathrm{H}_{4}\right)\right] \cdot \mathrm{H}_{2} \mathrm{O}$. (d) $\left[\mathrm{Nd}(4-\mathrm{ab})_{3}\left(\mathrm{~N}_{2} \mathrm{H}_{4}\right)\right] \cdot \mathrm{H}_{2} \mathrm{O}$. (e) $\left[\mathrm{Sm}(4-\mathrm{ab})_{3}\left(\mathrm{~N}_{2} \mathrm{H}_{4}\right)\right] \cdot \mathrm{H}_{2} \mathrm{O}$. (f) $\left[\mathrm{Gd}(4-\mathrm{ab})_{3}\left(\mathrm{~N}_{2} \mathrm{H}_{4}\right)\right] \cdot \mathrm{H}_{2} \mathrm{O}$.

TABLE 1: Analytical data of 3-abH and 4-abH complexes.

\begin{tabular}{|c|c|c|c|c|c|}
\hline \multicolumn{6}{|c|}{ Analytical data (\%) } \\
\hline Complexes & Carbon Fd (calc.) & Hydrogen Fd (calc.) & Nitrogen Fd (calc.) & Hydrazine Fd (calc.) & Metal Fd (calc.) \\
\hline$\left[\mathrm{La}(3-\mathrm{ab})_{3}\left(\mathrm{~N}_{2} \mathrm{H}_{4}\right)_{2}\right]$ & $43.6(43.8)$ & $4.1(3.9)$ & $7.3(7.6)$ & $8.4(8.6)$ & $18.7(18.8)$ \\
\hline$\left[\mathrm{Ce}(3-\mathrm{ab})_{3}\left(\mathrm{~N}_{2} \mathrm{H}_{4}\right)_{2}\right]$ & $44.0(43.7)$ & $4.0(3.9)$ & $7.4(7.6)$ & $8.5(8.6)$ & $18.7(18.9)$ \\
\hline$\left[\operatorname{Pr}(3-\mathrm{ab})_{3}\left(\mathrm{~N}_{2} \mathrm{H}_{4}\right)_{2}\right]$ & $43.1(43.6)$ & $4.0(3.9)$ & $7.4(7.5)$ & $8.7(8.6)$ & $18.9(19.0)$ \\
\hline$\left[\mathrm{Nd}(3-\mathrm{ab})_{3}\left(\mathrm{~N}_{2} \mathrm{H}_{4}\right)_{2}\right] \cdot \mathrm{H}_{2} \mathrm{O}$ & $42.3(42.4)$ & $4.0(4.1)$ & $7.3(7.3)$ & $8.3(8.4)$ & $18.9(18.9)$ \\
\hline$\left[\mathrm{Sm}(3-\mathrm{ab})_{3}\left(\mathrm{~N}_{2} \mathrm{H}_{4}\right)_{2}\right] \cdot \mathrm{H}_{2} \mathrm{O}$ & $42.2(42.1)$ & $4.0(4.1)$ & $7.2(7.3)$ & $8.3(8.3)$ & $19.5(19.5)$ \\
\hline$\left[\mathrm{Gd}(3-\mathrm{ab})_{3}\left(\mathrm{~N}_{2} \mathrm{H}_{4}\right)_{2}\right]$ & $43.0(42.7)$ & $3.7(3.9)$ & $7.3(7.4)$ & $8.5(8.4)$ & $20.7(20.7)$ \\
\hline$\left[\mathrm{La}(4-\mathrm{ab})_{3}\left(\mathrm{~N}_{2} \mathrm{H}_{4}\right)\right] \cdot \mathrm{H}_{2} \mathrm{O}$ & $44.5(44.6)$ & $3.7(3.8)$ & $4.0(3.9)$ & $4.4(4.4)$ & $19.5(19.1)$ \\
\hline$\left[\mathrm{Ce}(4-\mathrm{ab})_{3}\left(\mathrm{~N}_{2} \mathrm{H}_{4}\right)\right] \cdot \mathrm{H}_{2} \mathrm{O}$ & $44.5(44.5)$ & $3.6(3.7)$ & $3.8(3.9)$ & $4.4(4.4)$ & $19.2(19.3)$ \\
\hline$\left[\operatorname{Pr}(4-\mathrm{ab})_{3}\left(\mathrm{~N}_{2} \mathrm{H}_{4}\right)\right] \cdot \mathrm{H}_{2} \mathrm{O}$ & $45.0(44.5)$ & $3.7(3.7)$ & $3.7(3.8)$ & $4.4(4.4)$ & $19.5(19.4)$ \\
\hline$\left[\mathrm{Nd}(4-\mathrm{ab})_{3}\left(\mathrm{~N}_{2} \mathrm{H}_{4}\right)\right] \cdot \mathrm{H}_{2} \mathrm{O}$ & $44.0(44.3)$ & $3.6(3.7)$ & $3.7(3.8)$ & $4.4(4.4)$ & $19.6(19.7)$ \\
\hline$\left[\mathrm{Sm}(4-\mathrm{ab})_{3}\left(\mathrm{~N}_{2} \mathrm{H}_{4}\right)\right] \cdot \mathrm{H}_{2} \mathrm{O}$ & $43.8(43.9)$ & $3.5(3.7)$ & $3.7(3.8)$ & $4.3(4.3)$ & $20.6(20.4)$ \\
\hline$\left[\mathrm{Gd}(4-\mathrm{ab})_{3}\left(\mathrm{~N}_{2} \mathrm{H}_{4}\right)\right] \cdot \mathrm{H}_{2} \mathrm{O}$ & $43.1(43.5)$ & $4.1(3.9)$ & $3.9(3.8)$ & $4.3(4.3)$ & $21.4(21.1)$ \\
\hline
\end{tabular}




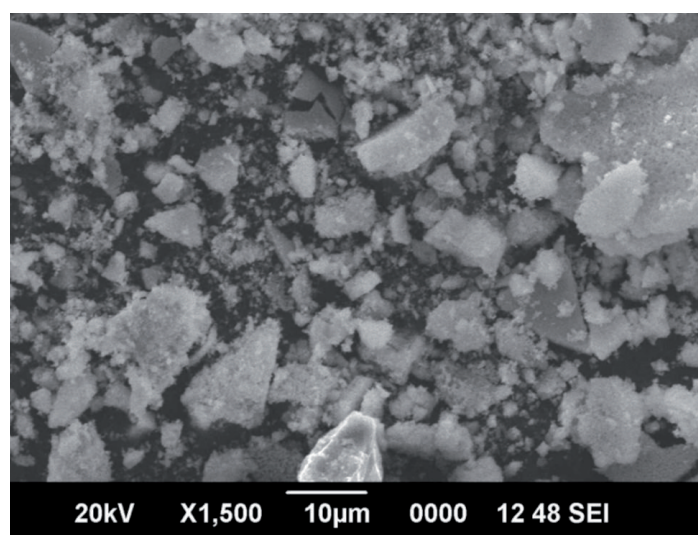

Figure 7: SEM image of $\mathrm{Gd}_{2} \mathrm{O}_{3}$-Obtained using [Gd(3$\mathrm{ab})_{3}\left(\mathrm{~N}_{2} \mathrm{H}_{4}\right)_{2}$ ] and the corresponding SEM-EDX image of $\mathrm{Gd}_{2} \mathrm{O}_{3}$ Shown in Figure 8.

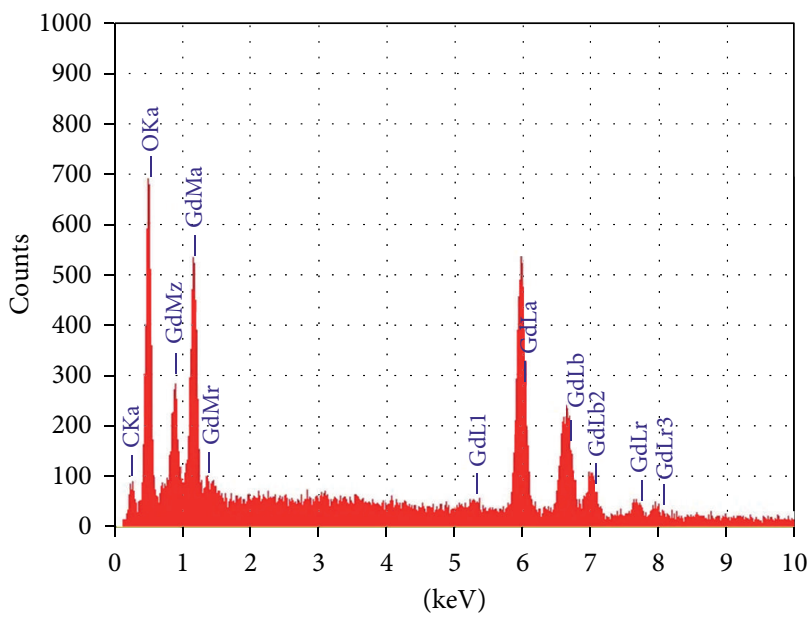

Figure 8: SEM-EDX image of $\mathrm{Gd}_{2} \mathrm{O}_{3}$.

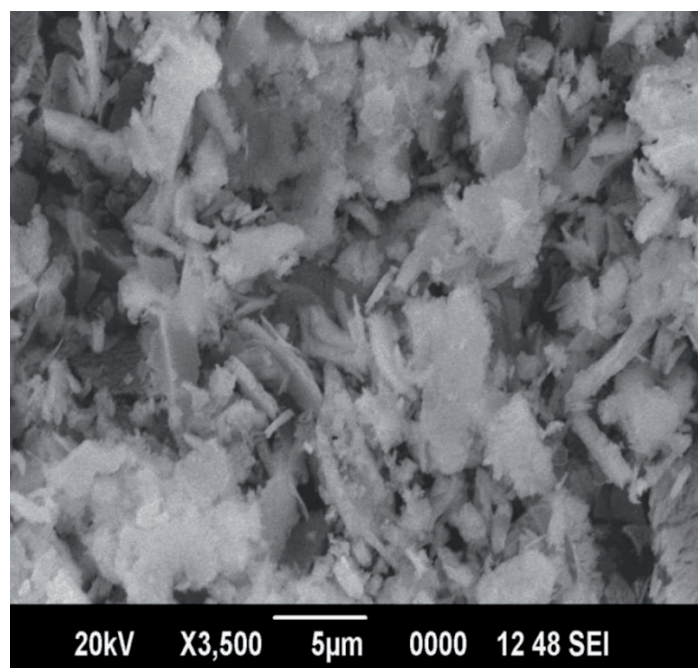

Figure 9: SEM image of $\mathrm{Nd}_{2} \mathrm{O}_{3}$-Obtained using [ $\mathrm{Nd}(4$ $\left.\mathrm{ab})_{3}\left(\mathrm{~N}_{2} \mathrm{H}_{4}\right)\right] \cdot \mathrm{H}_{2} \mathrm{O}$ and the corresponding SEM-EDX image of $\mathrm{Nd}_{2} \mathrm{O}_{3}$ Shown in Figure 10.

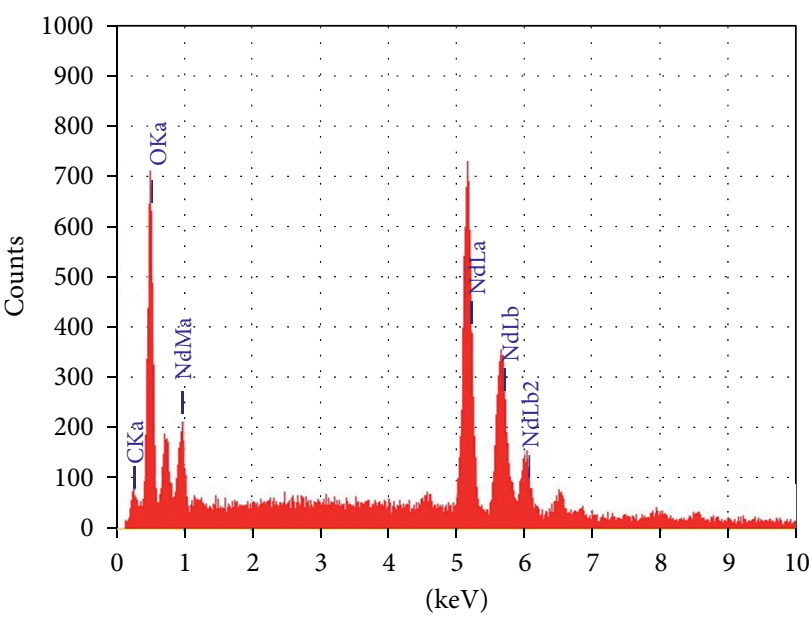

FIGURE 10: SEM-EDX image of $\mathrm{Nd}_{2} \mathrm{O}_{3}$.

TABLE 2: Electronic spectral data lanthanide complexes of 3-abH and $4-\mathrm{abH}$.

\begin{tabular}{|c|c|c|c|}
\hline Acid & Complex & Absorption maximum $\left(\mathrm{cm}^{-1}\right)$ & Assignment \\
\hline \multirow{5}{*}{ 3-abH } & \multirow{2}{*}{$\mathrm{Nd}^{+3}$} & 15552 & ${ }^{2} \mathrm{H}_{11 / 2}$ \\
\hline & & 12063 & ${ }^{3} \mathrm{H}_{9 / 2},{ }^{4} \mathrm{~S}_{3 / 2}$, \\
\hline & \multirow{3}{*}{$\operatorname{Pr}^{+3}$} & 15552 & ${ }^{2} \mathrm{H}_{11 / 2}$, \\
\hline & & 9208 & ${ }^{1} \mathrm{G}_{4}$ \\
\hline & & 7143 & ${ }^{3} \mathrm{~F}_{3},{ }^{3} \mathrm{~F}_{4}$ \\
\hline \multirow{11}{*}{ 4-abH } & \multirow{7}{*}{$\mathrm{Nd}^{+3}$} & 20000 & \multirow{7}{*}{$\begin{array}{c}{ }^{2} \mathrm{~F}_{3 / 2},{ }^{2} \mathrm{D}_{3 / 2} \\
{ }^{2} \mathrm{G}_{7 / 2} \\
{ }^{2} \mathrm{H}_{11 / 2} \\
{ }^{4} \mathrm{~F}_{7 / 2}, \\
{ }^{3} \mathrm{H}_{9 / 2},{ }^{4} \mathrm{~S}_{3 / 2} \\
{ }^{4} \mathrm{I}_{15 / 2},{ }^{4} \mathrm{I}_{15 / 2},\end{array}$} \\
\hline & & 17986 & \\
\hline & & 15673 & \\
\hline & & 13089 & \\
\hline & & 12005 & \\
\hline & & 6262 & \\
\hline & & 5714 & \\
\hline & \multirow{4}{*}{$\operatorname{Pr}^{+3}$} & 23310 & ${ }^{2} \mathrm{P}_{2}$ \\
\hline & & 17953 & ${ }^{1} \mathrm{D}_{2}$ \\
\hline & & 9588 & ${ }^{1} \mathrm{G}_{4}$ \\
\hline & & 7215 & ${ }^{3} \mathrm{~F}_{3},{ }^{3} \mathrm{~F}_{4}$ \\
\hline
\end{tabular}

preferably set in complexes with higher coordination number greater than eight, hydrazine molecules present in the complexes reported in this work should be of bridged bidental mode [20].

Similar to 3-isomer acid, 4-isomer acid also shows peaks at $1126 \mathrm{~cm}^{-1}, 1225 \mathrm{~cm}^{-1}$, and $1295 \mathrm{~cm}^{-1}$ due to $v_{\mathrm{C}-\mathrm{O} \text { ester }}$. The peak observed at $1753 \mathrm{~cm}^{-1}$ is due to $v_{\mathrm{C}=\mathrm{O} \text { ester }}$. The peak at $1680 \mathrm{~cm}^{-1}$ due to $v_{\mathrm{C}=\mathrm{O} \text { acid }}$ is found. In the case of complexes, $v_{\mathrm{C}-\mathrm{O} \text { ester }}$ is observed at $1157 \mathrm{~cm}^{-1}, 1285 \mathrm{~cm}^{-1}, 1326 \mathrm{~cm}^{-1}$, $1285 \mathrm{~cm}^{-1}, 1159 \mathrm{~cm}^{-1}$, and $1239 \mathrm{~cm}^{-1}$ for La, Ce, Pr, Nd, Sm and $\mathrm{Gd}$ complexes, respectively. Further $v_{\mathrm{C}=\mathrm{O} \text { acid }}$ found at $1680 \mathrm{~cm}^{-1}$ is split into two $v_{\mathrm{C}=\mathrm{O} \text { (asym) }}$ and $v_{\mathrm{C}=\mathrm{O}(\text { sym) }}$ peaks displaying at $1600 \mathrm{~cm}^{-1}$ (La), $1598 \mathrm{~cm}^{-1}$ (Ce), $1601 \mathrm{~cm}^{-1}$ (Pr), $1601 \mathrm{~cm}^{-1}$ (Nd), $1599 \mathrm{~cm}^{-1}(\mathrm{Sm}), 1600 \mathrm{~cm}^{-1}$ (Gd) and $1404 \mathrm{~cm}^{-1}$ (La), $1417 \mathrm{~cm}^{-1}$ (Ce), $1406 \mathrm{~cm}^{-1}$ (Pr), $1417 \mathrm{~cm}^{-1}$ 
TABLE 3: IR data of lanthanide complexes of 3-abH and 4-abH (4-abH: 4-acetoxy benzoic acid, 3-abH: 3-acetoxy benzoic acid).

\begin{tabular}{|c|c|c|c|c|c|c|c|c|c|c|c|}
\hline Acid & $\begin{array}{c}v_{\mathrm{C}-\mathrm{O} \text { ester }} \\
\mathrm{cm}^{-1}\end{array}$ & $\begin{array}{c}v_{\mathrm{C}=\mathrm{O} \text { ester }} \\
\mathrm{cm}^{-1}\end{array}$ & $\begin{array}{c}v_{\mathrm{C}=\mathrm{O} \text { acid }} \\
\mathrm{cm}^{-1}\end{array}$ & Complexes & $\begin{array}{c}v_{\mathrm{C}=\mathrm{O} \text { asym }} \\
\mathrm{cm}^{-1}\end{array}$ & $\begin{array}{c}v_{\mathrm{C}=\mathrm{O} \text { sym }} \\
\mathrm{cm}^{-1}\end{array}$ & $\begin{array}{l}v_{\mathrm{N}-\mathrm{N}} \\
\mathrm{cm}^{-1}\end{array}$ & $\begin{array}{l}v_{\mathrm{N}-\mathrm{H}} \\
\mathrm{cm}^{-1}\end{array}$ & $\begin{array}{l}v_{\mathrm{O}-\mathrm{H}} \\
\mathrm{cm}^{-1}\end{array}$ & $\begin{array}{c}v_{\mathrm{HOH} \text { bending }} \\
\mathrm{cm}^{-1}\end{array}$ & $\begin{array}{c}v_{\mathrm{C}-\mathrm{O} \text { ester }} \\
\mathrm{cm}^{-1}\end{array}$ \\
\hline \multirow{6}{*}{$3-\mathrm{abH}$} & \multirow{6}{*}{$\begin{array}{l}1091 \mathrm{~m}, \\
1207 \mathrm{~s}, \\
1276 \mathrm{~s}\end{array}$} & \multirow{6}{*}{$1761 \mathrm{~s}$} & \multirow{6}{*}{$1676 s$} & {$\left[\mathrm{La}(3-\mathrm{ab})_{3}\left(\mathrm{~N}_{2} \mathrm{H}_{4}\right)_{2}\right]$} & $1602 \mathrm{~s}$ & $1433 \mathrm{~m}$ & $\begin{array}{l}937 \mathrm{~s} \\
1001 \mathrm{~s}\end{array}$ & $3358 \mathrm{~b}$ & - & & 1315 \\
\hline & & & & {$\left[\mathrm{Ce}(3-\mathrm{ab})_{3}\left(\mathrm{~N}_{2} \mathrm{H}_{4}\right)_{2}\right]$} & $1599 \mathrm{~s}$ & $1430 \mathrm{~b}$ & $\begin{array}{l}962 \mathrm{~s} \\
1003 \mathrm{~m}\end{array}$ & $3273 b$ & - & & 1277 \\
\hline & & & & $\left.\left[\operatorname{Pr}(3-\mathrm{ab})_{3} \mathrm{~N}_{2} \mathrm{H}_{4}\right)_{2}\right]$ & $1587 \mathrm{~s}$ & $1410 \mathrm{~b}$ & $\begin{array}{c}929 \mathrm{~s} \\
1003 \mathrm{~b}\end{array}$ & $3373 b$ & - & & 1300 \\
\hline & & & & {$\left[\mathrm{Nd}(3-\mathrm{ab})_{3}\left(\mathrm{~N}_{2} \mathrm{H}_{4}\right)_{2}\right] \cdot \mathrm{H}_{2} \mathrm{O}$} & $1595 \mathrm{~s}$ & $1429 \mathrm{~m}$ & $\begin{array}{c}931 \mathrm{~s} \\
1001 \mathrm{~m}\end{array}$ & $3277 \mathrm{~b}$ & $3335 b$ & $862 \mathrm{~m}$ & 1303 \\
\hline & & & & {$\left[\mathrm{Sm}(3-\mathrm{ab})_{3}\left(\mathrm{~N}_{2} \mathrm{H}_{4}\right)_{2}\right] \cdot \mathrm{H}_{2} \mathrm{O}$} & $1589 \mathrm{~s}$ & $1433 \mathrm{~m}$ & $\begin{array}{c}931 \mathrm{~s} \\
1005 \mathrm{~m}\end{array}$ & $3300 \mathrm{~b}$ & $3300 \mathrm{~b}$ & $820 \mathrm{~s}$ & 1300 \\
\hline & & & & {$\left[\mathrm{Gd}(3-\mathrm{ab})_{3}\left(\mathrm{~N}_{2} \mathrm{H}_{4}\right)_{2}\right]$} & $1593 \mathrm{~s}$ & $1430 \mathrm{~m}$ & $\begin{array}{c}924 \mathrm{~s} \\
1005 \mathrm{~m}\end{array}$ & $3268 \mathrm{~b}$ & - & - & 1300 \\
\hline \multirow{6}{*}{$4-\mathrm{abH}$} & \multirow{6}{*}{$\begin{array}{l}1126 \mathrm{~s}, \\
1225 \mathrm{~b}, \\
1290 \mathrm{~m}\end{array}$} & \multirow{6}{*}{$1753 \mathrm{~s}$} & \multirow{6}{*}{$1683 b$} & {$\left[\mathrm{La}(4-\mathrm{ab})_{3}\left(\mathrm{~N}_{2} \mathrm{H}_{4}\right)\right] \cdot \mathrm{H}_{2} \mathrm{O}$} & $1600 \mathrm{~m}$ & $1404 \mathrm{~b}$ & $932 \mathrm{~s}$ & $3330 \mathrm{~b}$ & $3380 \mathrm{~b}$ & $845 \mathrm{~s}$ & $1157 \mathrm{~m}$ \\
\hline & & & & {$\left[\mathrm{Ce}(4-\mathrm{ab})_{3}\left(\mathrm{~N}_{2} \mathrm{H}_{4}\right)\right] \cdot \mathrm{H}_{2} \mathrm{O}$} & $1598 \mathrm{~s}$ & $1417 \mathrm{~s}$ & $929 \mathrm{~s}$ & $3200 \mathrm{~m}$ & $3379 \mathrm{~s}$ & $847 \mathrm{~s}$ & $1285 \mathrm{~s}$ \\
\hline & & & & {$\left[\operatorname{Pr}(4-\mathrm{ab})_{3}\left(\mathrm{~N}_{2} \mathrm{H}_{4}\right)\right] \cdot \mathrm{H}_{2} \mathrm{O}$} & $1601 \mathrm{~s}$ & $1406 \mathrm{~b}$ & $932 \mathrm{~s}$ & $3276 b$ & $3343 \mathrm{~m}$ & $848 \mathrm{~s}$ & $1326 s$ \\
\hline & & & & {$\left[\mathrm{Nd}(4-\mathrm{ab})_{3}\left(\mathrm{~N}_{2} \mathrm{H}_{4}\right)\right] \cdot \mathrm{H}_{2} \mathrm{O}$} & $1601 \mathrm{~m}$ & $1415 b$ & $932 \mathrm{~s}$ & $3294 b$ & $3374 \mathrm{~b}$ & $849 \mathrm{~s}$ & $1285 \mathrm{~m}$ \\
\hline & & & & {$\left[\mathrm{Sm}(4-\mathrm{ab})_{3}\left(\mathrm{~N}_{2} \mathrm{H}_{4}\right)\right] \cdot \mathrm{H}_{2} \mathrm{O}$} & $1599 \mathrm{~s}$ & $1416 \mathrm{~m}$ & $931 \mathrm{~s}$ & $3391 \mathrm{~b}$ & $3390 \mathrm{~b}$ & $845 s$ & $1159 \mathrm{~s}$ \\
\hline & & & & {$\left[\mathrm{Gd}(4-\mathrm{ab})_{3}\left(\mathrm{~N}_{2} \mathrm{H}_{4}\right)\right] \cdot \mathrm{H}_{2} \mathrm{O}$} & $1600 s$ & $1417 \mathrm{~s}$ & $930 \mathrm{~s}$ & $3364 \mathrm{~b}$ & $3396 b$ & $847 \mathrm{~s}$ & $1239 \mathrm{~m}$ \\
\hline
\end{tabular}

s: sharp, m: medium, b: broad.

(Gd), $1415 \mathrm{~cm}^{-1}(\mathrm{Nd})$, and $1416 \mathrm{~cm}^{-1}(\mathrm{Sm})$. The difference $\Delta v$ being less than $200 \mathrm{~cm}^{-1}$ implies the bidental coordination. The sharp peaks observed in the range of $929-932 \mathrm{~cm}^{-1}$ are assigned to $\mathrm{N}-\mathrm{N}$ stretching of hydrazine which is involved in bidental coordination with the metal ion.

In addition, the hydrated complexes show broadbands observed in the range of $3400 \mathrm{~cm}^{-1}$ corresponding to $\mathrm{OH}$ stretching and $862 \mathrm{~cm}^{-1}$ corresponding to $\mathrm{OH}$ bending of water molecules present in the lattice. This broadband extends up to $3030 \mathrm{~cm}^{-1}$ may be because of the merging of $\mathrm{N}-\mathrm{H}$ stretching of hydrazine molecule with $v_{\mathrm{O}-\mathrm{H}}$ of water.

3.3. Thermal Data of Complexes. The thermal data of the complexes were given in Table 4.

3.3.1. Thermal Analysis of Lanthanides of 3-abH. The lanthanides of $3-\mathrm{abH}$ show two steps of decomposition in their TG curve, namely, dehydrazination and decomposition to their corresponding metal oxides without forming intermediates. Among them, Sm and Nd complexes being hydrated show endothermic dehydration in the temperature range of $40-45^{\circ} \mathrm{C}$ showing the weight loss temperature of $30-100^{\circ} \mathrm{C}$ in the TG. All the complexes show exothermic dehydrazination between 180 and $197^{\circ} \mathrm{C}$ and undergo oxidative decomposition to form their corresponding metal oxides, displaying the exotherms in the range of $437-640^{\circ} \mathrm{C}$ in DTA.

The TG-DTA of $\left[\operatorname{Pr}(3-\mathrm{ab})_{3}\left(\mathrm{~N}_{2} \mathrm{H}_{4}\right)_{2}\right], \quad[\mathrm{Sm}(3-$ $\left.\mathrm{ab})_{3}\left(\mathrm{~N}_{2} \mathrm{H}_{4}\right)_{2}\right] \cdot \mathrm{H}_{2} \mathrm{O}$, and $\left[\mathrm{Pr}(4-\mathrm{ab})_{3}\left(\mathrm{~N}_{2} \mathrm{H}_{4}\right)\right] \cdot \mathrm{H}_{2} \mathrm{O}$, $[\mathrm{Sm}(4-$ ab) $\left.{ }_{3}\left(\mathrm{~N}_{2} \mathrm{H}_{4}\right)\right] \cdot \mathrm{H}_{2} \mathrm{O}$ are shown in Figures $1,2,3$, and 4 as representative examples.
The sequence of reactions proposed for the decompositions is:

$$
\begin{aligned}
& {\left[\operatorname{Ln}\left\{\left(\mathrm{C}_{6} \mathrm{H}_{4}\left(\mathrm{OCOCH}_{3}\right) \mathrm{COO}\right)_{3}\right\}\left(\mathrm{N}_{2} \mathrm{H}_{4}\right)_{2}\right] \cdot \mathrm{H}_{2} \mathrm{O}} \\
& \stackrel{-\mathrm{H}_{2} \mathrm{O}}{\longrightarrow}\left[\operatorname{Ln}\left\{\left(\mathrm{C}_{6} \mathrm{H}_{4}\left(\mathrm{OCOCH}_{3}\right) \mathrm{COO}\right)_{3}\right\}\left(\mathrm{N}_{2} \mathrm{H}_{4}\right)_{2}\right],
\end{aligned}
$$

where $\mathrm{Ln}=\mathrm{Sm}$ and $\mathrm{Nd}$

$$
\begin{aligned}
& {\left[\operatorname{Ln}\left\{\left(\mathrm{C}_{6} \mathrm{H}_{4}\left(\mathrm{OCOCH}_{3}\right) \mathrm{COO}\right)_{3}\right\}\left(\mathrm{N}_{2} \mathrm{H}_{4}\right)_{2}\right]} \\
& \quad \stackrel{180-197^{\circ} \mathrm{C}}{\longrightarrow-2 \mathrm{~N}_{2} \mathrm{H}_{4}}\left[\operatorname{Ln}\left\{\left(\mathrm{C}_{6} \mathrm{H}_{4}\left(\mathrm{OCOCH}_{3}\right) \mathrm{COO}\right)_{3}\right\}\right] \\
& \stackrel{474-640^{\circ} \mathrm{C}}{\longrightarrow} \mathrm{Ln}_{2} \mathrm{O}_{3},
\end{aligned}
$$

where $\mathrm{Ln}=\mathrm{Ln}, \operatorname{Pr}$ and $\mathrm{Gd}$

$$
\begin{aligned}
& {\left[\mathrm{Ce}\left\{\left(\mathrm{C}_{6} \mathrm{H}_{4}\left(\mathrm{OCOCH}_{3}\right) \mathrm{COO}\right)_{3}\right\}\left(\mathrm{N}_{2} \mathrm{H}_{4}\right)_{2}\right]} \\
& \quad \stackrel{191^{\circ} \mathrm{C}}{\longrightarrow-2 \mathrm{~N}_{2} \mathrm{H}_{4}}\left[\mathrm{Ce}\left\{\left(\mathrm{C}_{6} \mathrm{H}_{4}\left(\mathrm{OCOCH}_{3}\right) \mathrm{COO}\right)_{3}\right\}\right] \\
& \quad \stackrel{437^{\circ} \mathrm{C}}{\longrightarrow} \mathrm{CeO}_{2} .
\end{aligned}
$$

where $\mathrm{Ln}=\mathrm{Ln}, \mathrm{Pr}$ and Gd

3.3.2. Thermal Analysis of Lanthanides of 4-abH. La, Pr, $\mathrm{Nd}$, and Sm complexes with 4-abH show almost similar type of decomposition pattern in their thermograms. They undergo dehydration showing endotherms in the range of $50-71^{\circ} \mathrm{C}$, dehydrazination showing exotherms in the range 
TABLE 4: Thermal data of lanthanide complexes of 3-abH and 4-abH.

\begin{tabular}{|c|c|c|c|c|c|}
\hline \multirow{3}{*}{ Complex } & \multirow{3}{*}{ DTA Temp $\left({ }^{\circ} \mathrm{C}\right)$} & \multicolumn{3}{|c|}{ Thermo gravimetry } & \multirow{3}{*}{ Nature of the reaction } \\
\hline & & \multirow{2}{*}{ Temp. Range $\left({ }^{\circ} \mathrm{C}\right)$} & \multicolumn{2}{|c|}{ Weight Loss \% } & \\
\hline & & & Obsd. & Calcd. & \\
\hline \multirow{4}{*}[\mathrm{La}(3-\mathrm{ab})_{3}(\mathrm{N}_{2}\mathrm{H}_{4})_{2}]{} & $180.0(-)$ & $60-120$ & 8.5 & 8.6 & Dehydrazination \\
\hline & $406.0(-) \mathrm{s}$ & & & & \\
\hline & $510.0(-) b$ & $120-700$ & 78.1 & 78.0 & Decomposition to metal oxide \\
\hline & $640.0(-)$ & & & & \\
\hline \multirow{3}{*}[\mathrm{Ce}(3-\mathrm{ab})_{3}(\mathrm{N}_{2}\mathrm{H}_{4})_{2}]{} & $191.4(-)$ & $120-200$ & & & \\
\hline & $226.0(-) \mathrm{d}$ & $200-280$ & 8.5 & 8.6 & Dehydrazination \\
\hline & $437.0(-) \mathrm{s}$ & $280-600$ & 76.5 & 76.7 & Decomposition to metal oxide \\
\hline \multirow{4}{*}[\operatorname{Pr}(3-\mathrm{ab})_{3}(\mathrm{N}_{2}\mathrm{H}_{4})_{2}]{} & $190.0(-) \mathrm{s}$ & $150-195$ & 8.7 & 8.6 & Dehydrazination \\
\hline & $200.0(-)$ & & & & \\
\hline & $289.0(-)$ & $195-700$ & 77.0 & 77.0 & Decomposition to metal oxide \\
\hline & $477.0(-) \mathrm{s}$ & & & & \\
\hline \multirow{4}{*}[\mathrm{Nd}(3-\mathrm{ab})_{3}(\mathrm{N}_{2}\mathrm{H}_{4})_{2}]{$\cdot \mathrm{H}_{2} \mathrm{O}$} & $40.0(+)$ & $30-100$ & 2.2 & 2.4 & Dehydration \\
\hline & $197.4(-) \mathrm{s}$ & $100-200$ & 10.6 & 10.7 & Dehydrazination \\
\hline & $248.6(-) b$ & $200-700$ & 78.0 & 77.9 & Decomposition to metal oxide \\
\hline & $474.3(-) \mathrm{s}$ & & & & \\
\hline \multirow{4}{*}[\mathrm{Sm}(3-\mathrm{ab})_{3}(\mathrm{N}_{2}\mathrm{H}_{4})_{2}]{$\cdot \mathrm{H}_{2} \mathrm{O}$} & $45.0(+)$ & $30-100$ & 2.4 & 2.3 & Dehydration \\
\hline & $186.0(-)$ & $100-190$ & 10.5 & 10.6 & Dehydrazination \\
\hline & $240.0(-) b$ & $190-700$ & 77.4 & 77.3 & Formation of metal oxide \\
\hline & $480.0(-) \mathrm{s}$ & (1) & . & 每 & 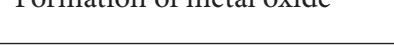 \\
\hline \multirow{4}{*}[\mathrm{Gd}(3-\mathrm{ab})_{3}(\mathrm{N}_{2}\mathrm{H}_{4})_{2}]{} & $186.0(-)$ & $160-210$ & 8.5 & 8.4 & Dehydrazination \\
\hline & $226.0(-) \mathrm{d}$ & & & & \\
\hline & $320.0(-)$ & $210-700$ & 76.2 & 76.1 & Decomposition to metal oxide \\
\hline & $474.2(-) \mathrm{s}$ & & & & \\
\hline \multirow{6}{*}[\mathrm{La}(4-\mathrm{ab})_{3}(\mathrm{N}_{2}\mathrm{H}_{4})]{$\cdot \mathrm{H}_{2} \mathrm{O}$} & $60.3(+)$ & $40-100$ & 2.3 & 2.5 & Dehydration \\
\hline & $166.0(-)$ & $100-180$ & 6.9 & 6.9 & Dehydrazination \\
\hline & $247.0(-) \mathrm{s}$ & $180-265$ & 62.1 & 62.0 & Formation of metal carbonate \\
\hline & $323.0(-)$ & & & & \\
\hline & $475.1(-)$ & $265-700$ & 77.2 & 77.6 & Decomposition to metal oxide \\
\hline & $543.0(-) b$ & & & & \\
\hline \multirow{5}{*}[\mathrm{Ce}(4-\mathrm{ab})_{3}(\mathrm{N}_{2}\mathrm{H}_{4})]{$\cdot \mathrm{H}_{2} \mathrm{O}$} & $50.0(+) b$ & $80-190$ & 2.3 & 2.5 & Dehydration \\
\hline & $225.0(-)$ & & & & \\
\hline & $323.0(-) \mathrm{s}$ & $190-700$ & 76.1 & 76.3 & Decomposition to metal oxide \\
\hline & $418.0(-)$ & & & & \\
\hline & $490.0(-) b$ & & & & \\
\hline \multirow{6}{*}[\operatorname{Pr}(4-\mathrm{ab})_{3}(\mathrm{N}_{2}\mathrm{H}_{4})]{$\cdot \mathrm{H}_{2} \mathrm{O}$} & $71.2(+)$ & $40-100$ & 2.6 & 2.5 & Dehydration \\
\hline & $167.0(-)$ & $100-175$ & 7.0 & 6.9 & Dehydrazination \\
\hline & $200.0(-)$ & $175-440(-)$ & 61.5 & 61.4 & Formation of metal carbonate \\
\hline & 275.0 & & & & \\
\hline & $475.0(-)$ & $440-700$ & 76.9 & 767 & Decomposition to metal oxide \\
\hline & $541.8(-) \mathrm{s}$ & & & & \\
\hline \multirow{6}{*}[\mathrm{Nd}(4-\mathrm{ab})_{3}(\mathrm{N}_{2}\mathrm{H}_{4})]{$\cdot \mathrm{H}_{2} \mathrm{O}$} & $60.5(+)$ & $40-100$ & 2.3 & 2.5 & Dehydration \\
\hline & $167.2(-)$ & $100-190$ & 6.9 & 6.8 & Dehydrazination \\
\hline & $205.0(-)$ & & & & \\
\hline & $247.0(-)$ & $190-700$ & 77.2 & 77.0 & Decomposition to metal oxide \\
\hline & $387.0(-)$ & & & & \\
\hline & $540.2(-) \mathrm{s}$ & & & & \\
\hline
\end{tabular}


TABLe 4: Continued.

\begin{tabular}{|c|c|c|c|c|c|}
\hline \multirow{3}{*}{ Complex } & \multirow{3}{*}{ DTA Temp $\left({ }^{\circ} \mathrm{C}\right)$} & \multicolumn{3}{|c|}{ Thermo gravimetry } & \multirow{3}{*}{ Nature of the reaction } \\
\hline & & \multirow{2}{*}{ Temp. Range $\left({ }^{\circ} \mathrm{C}\right)$} & \multicolumn{2}{|c|}{ Weight Loss \% } & \\
\hline & & & Obsd. & Calcd. & \\
\hline \multirow{6}{*}[\mathrm{Sm}(4-\mathrm{ab})_{3}(\mathrm{N}_{2}\mathrm{H}_{4})]{$\cdot \mathrm{H}_{2} \mathrm{O}$} & $62.4(+)$ & $40-150$ & 2.5 & 2.4 & Dehydration \\
\hline & $175.3(-)$ & $150-200$ & 6.7 & 6.8 & Dehydrazination \\
\hline & $213.2(-)$ & \multirow{4}{*}{$200-700$} & \multirow{4}{*}{76.3} & \multirow{4}{*}{76.4} & \multirow{4}{*}{ Decomposition to metal oxide } \\
\hline & $247.0(-)$ & & & & \\
\hline & $481.3(+)$ & & & & \\
\hline & $550.0(-) \mathrm{b}$ & & & & \\
\hline \multirow{5}{*}[\mathrm{Gd}(4-\mathrm{ab})_{3}(\mathrm{N}_{2}\mathrm{H}_{4})]{$\cdot \mathrm{H}_{2} \mathrm{O}$} & $63.2(+)$ & $40-150$ & 2.2 & 2.4 & Dehydration \\
\hline & $178.0(-)$ & \multirow{4}{*}{$150-700$} & \multirow{4}{*}{75.7} & \multirow{4}{*}{75.5} & \multirow{4}{*}{ Decomposition to metal oxide } \\
\hline & $275.0(-)$ & & & & \\
\hline & $448.1(-)$ & & & & \\
\hline & $549.0(-)$ & & & & \\
\hline
\end{tabular}

(+): endo, (-): exo, s: singlet, d: doublet, b: broad.

TABLE 5: X-ray diffraction data of lanthanide complexes of 3-abH and 4-abH (D spacing in $\AA$ units and intensity in parentheses).

\begin{tabular}{|c|c|c|c|c|c|}
\hline \multicolumn{6}{|c|}{$\left[\operatorname{Ln}(3-\mathrm{ab})_{3}\left(\mathrm{~N}_{2} \mathrm{H}_{4}\right)_{2}\right] \cdot x \mathrm{H}_{2} \mathrm{O}$} \\
\hline $\mathrm{La}$ & $\mathrm{Ce}$ & $\operatorname{Pr}$ & $\mathrm{Nd}$ & $\mathrm{Sm}$ & Gd \\
\hline $11.5531(10.90)$ & $9.9750(15.40)$ & $9.7115(21.80)$ & $9.7427(24.70)$ & $9.6352(15.70)$ & $9.6122(10.00)$ \\
\hline $10.2367(7.30)$ & $9.5507(25.00)$ & & & & $6.3314(21.70)$ \\
\hline $6.5266(14.50)$ & $6.2677(59.60)$ & $6.3777(43.60)$ & $6.3563(35.60)$ & $6.3011(21.40)$ & $4.8156(21.70)$ \\
\hline $5.3813(4.00)$ & $5.2698(9.60)$ & $5.3390(5.50)$ & $4.8651(35.60)$ & $4.8100(31.40)$ & $4.1634(13.30)$ \\
\hline $5.1392(4.50)$ & $4.9985(48.38)$ & $5.0584(20.00)$ & $4.1932(19.20)$ & $4.1718(31.40)$ & $3.8362(38.30)$ \\
\hline $4.6080(38.20)$ & $4.7902(40.40)$ & $4.8561(40.00)$ & $3.8369(43.80)$ & $3.8350(42.90)$ & \\
\hline 4.1537 (10.90) & $4.2796(40.40)$ & $4.1857(25.50)$ & $3.4426(11.00)$ & $3.5184(12.90)$ & \\
\hline $3.7752(3.40)$ & $4.1427(40.40)$ & $3.8368(52.73)$ & $3.2630(100.00)$ & $3.2532(100.00)$ & \\
\hline $3.5802(21.80)$ & $3.8030(48.10)$ & $3.5400(25.50)$ & $2.7969(5.50)$ & $2.4051(10.00)$ & \\
\hline $3.3916(34.50)$ & $3.5070(25.00)$ & $3.3826(14.50)$ & $2.5720(1.40)$ & $2.2485(7.10)$ & \\
\hline $3.1643(34.50)$ & $3.3427(7.70)$ & $3.2571(100.00)$ & & & \\
\hline $2.9345(16.40)$ & $3.2274(100.00)$ & $3.0248(10.90)$ & & & \\
\hline $2.2874(12.70)$ & $2.7711(3.80)$ & $2.4107(14.50)$ & & & \\
\hline \multicolumn{6}{|c|}{$\left[\operatorname{Ln}(4-\mathrm{ab})_{3}\left(\mathrm{~N}_{2} \mathrm{H}_{4}\right)\right] \cdot \mathrm{H}_{2} \mathrm{O}$} \\
\hline \multicolumn{6}{|c|}{ where Ln: La, Ce, Pr, Nd, Sm, and Gd, and 4-abH: 4-acetoxy benzoic acid } \\
\hline $\mathrm{La}$ & $\mathrm{Ce}$ & $\operatorname{Pr}$ & $\mathrm{Nd}$ & $\mathrm{Sm}$ & Gd \\
\hline $10.3780(52.10)$ & & $10.3974(80.40)$ & $10.2188(29.40)$ & $10.5230(23.60)$ & $10.3142(21.70)$ \\
\hline $5.0111(79.20)$ & $5.0852(25.83)$ & $9.0799(100.0)$ & $8.8619(33.00)$ & $9.5067(20.00)$ & $9.5467(36.70)$ \\
\hline $4.5658(43.80)$ & $4.5507(24.49)$ & $5.0283(67.40)$ & $6.5901(13.70)$ & $6.3442(20.00)$ & $5.0142(45.00)$ \\
\hline $3.9225(39.60)$ & $3.6554(100.0)$ & $4.5186(60.90)$ & $5.5530(15.70)$ & $5.0280(74.50)$ & $4.5436(28.30)$ \\
\hline $3.3174(72.90)$ & $3.3221(42.61)$ & $3.9491(84.80)$ & $4.9752(25.50)$ & $4.5902(18.20)$ & $3.9100(23.30)$ \\
\hline $3.1956(35.40)$ & $3.0444(14.16)$ & $3.4136(43.50)$ & $3.5742(100.00)$ & $3.8450(18.20)$ & $3.4281(25.00)$ \\
\hline 2.9905 (77.10) & $2.9899(83.93)$ & $3.1655(73.90)$ & $3.2815(17.60)$ & $3.4495(21.80)$ & $3.3191(50.00)$ \\
\hline $2.5716(31.30)$ & $2.5720(3.25)$ & $2.9805(67.40)$ & $3.1302(15.70)$ & $3.3172(47.30)$ & $2.9848(60.00)$ \\
\hline $2.4191(29.20)$ & $2.4120(9.26)$ & $2.5601(65.20)$ & $2.9494(19.60)$ & $2.9754(100.0)$ & $2.6726(23.30)$ \\
\hline $2.1659(37.50)$ & $2.1779(3.96)$ & $2.4124(45.70)$ & $2.5377(13.73)$ & $2.6822(18.20)$ & $2.4047(25.00)$ \\
\hline $1.5059(14.60)$ & & $2.1176(41.30)$ & $2.4634(11.76)$ & $2.4916(34.50)$ & $2.1502(20.00)$ \\
\hline \multirow[t]{2}{*}{$12450(18.80)$} & $1.5775(0.88)$ & $1.5186(32.60)$ & & $2.2736(18.20)$ & \\
\hline & & & & $1.5759(29.10)$ & $1.2850(13.30)$ \\
\hline
\end{tabular}


of $166-175^{\circ} \mathrm{C}$ and oxidative decomposition showing many exotherms in the range of $213-550^{\circ} \mathrm{C}$ to metal oxide residue. $\mathrm{La}$ and Pr complexes show the formation of metal carbonate intermediate [21] before final decomposition to metal oxide, whereas the other two do not.

Ce and Gd complexes, though found to follow similar type of decomposition to form metal oxide residue, the TG traces are not clear to identify dehydrazination and the intermediate formation. The thermal degradation patterns are indicated by the following reactions:

$$
\begin{aligned}
& {\left[\operatorname{Ln}\left\{\left(\mathrm{C}_{6} \mathrm{H}_{4}\left(\mathrm{OCOCH}_{3}\right) \mathrm{COO}\right)_{3}\right\}\left(\mathrm{N}_{2} \mathrm{H}_{4}\right)\right] \cdot \mathrm{H}_{2} \mathrm{O}} \\
& \stackrel{-50-71^{\circ} \mathrm{C}}{\longrightarrow}\left[\operatorname{Ln}\left\{\left(\mathrm{C}_{6} \mathrm{H}_{4}\left(\mathrm{OCOCH}_{3}\right) \mathrm{COO}\right)_{3}\right\}\left(\mathrm{N}_{2} \mathrm{H}_{4}\right)\right],
\end{aligned}
$$

where $\mathrm{Ln}=\mathrm{La}, \mathrm{Ce}, \mathrm{Pr}, \mathrm{Nd}, \mathrm{Sm}$ and Gd

$$
\begin{aligned}
& {\left[\operatorname{Ln}\left\{\left(\mathrm{C}_{6} \mathrm{H}_{4}\left(\mathrm{OCOCH}_{3}\right) \mathrm{COO}\right)_{3}\right\}\left(\mathrm{N}_{2} \mathrm{H}_{4}\right)\right]} \\
& \stackrel{-166-175^{\circ} \mathrm{C}}{\underset{-\mathrm{N}_{2} \mathrm{H}_{4}}{\longrightarrow}}\left[\operatorname{Ln}\left\{\left(\mathrm{C}_{6} \mathrm{H}_{4}\left(\mathrm{OCOCH}_{3}\right) \mathrm{COO}\right)_{3}\right\}\right],
\end{aligned}
$$

where $\mathrm{Ln}=\mathrm{La}, \mathrm{Pr}, \mathrm{Nd}$ and $\mathrm{Sm}$

$$
\begin{aligned}
& {\left[\mathrm{Ln}\left\{\left(\mathrm{C}_{6} \mathrm{H}_{4}\left(\mathrm{OCOCH}_{3}\right) \mathrm{COO}\right)_{3}\right\}\right]} \\
& \stackrel{200-323^{\circ} \mathrm{C}}{\longrightarrow} \mathrm{Ln}_{2}\left(\mathrm{CO}_{3}\right)_{3} \stackrel{475-543^{\circ} \mathrm{C}}{\longrightarrow} \mathrm{Ln}_{2} \mathrm{O}_{3},
\end{aligned}
$$

where $\mathrm{Ln}=\mathrm{La}$ and $\mathrm{Pr}$

$$
\left[\mathrm{Ln}\left\{\left(\mathrm{C}_{6} \mathrm{H}_{4}\left(\mathrm{OCOCH}_{3}\right) \mathrm{COO}\right)_{3}\right\}\right] \stackrel{540-550^{\circ} \mathrm{C}}{\longrightarrow} \mathrm{Ln}_{2} \mathrm{O}_{3} \text {, }
$$

where $\mathrm{Ln}=\mathrm{Nd}$ and $\mathrm{Sm}$

$$
\begin{gathered}
{\left[\mathrm{Ce}\left\{\left(\mathrm{C}_{6} \mathrm{H}_{4}\left(\mathrm{OCOCH}_{3}\right) \mathrm{COO}\right)_{3}\right\}\left(\mathrm{N}_{2} \mathrm{H}_{4}\right)\right] \stackrel{490^{\circ} \mathrm{C}}{\longrightarrow} \mathrm{CeO}_{2}} \\
{\left[\mathrm{Gd}\left\{\left(\mathrm{C}_{6} \mathrm{H}_{4}\left(\mathrm{OCOCH}_{3}\right) \mathrm{COO}\right)_{3}\right\}\left(\mathrm{N}_{2} \mathrm{H}_{4}\right)\right] \stackrel{549^{\circ} \mathrm{C}}{\longrightarrow} \mathrm{Gd}_{2} \mathrm{O}_{3} .}
\end{gathered}
$$

3.4. X-Ray Diffraction Analysis. The powder XRD patterns along with their $d$ spacings are given in Table 5 . The comparison of XRD patterns of the lanthanide complexes is shown in Figures 5 and 6 . The XRD patterns of lanthanides of $4-\mathrm{abH}$ and $3-\mathrm{abH}$ reveal that each set of complexes has similarity in their structures implying similar compositions.

3.5. SEM-EDX Studies. The complexes were calcined in muffle furnace at their decomposition temperature, heating subsequently at the same temperature, and analyzed for their morphology and particle size. The SEM-EDX images of residues obtained from $\left[\mathrm{Gd}(3-\mathrm{ab})_{3}\left(\mathrm{~N}_{2} \mathrm{H}_{4}\right)_{2}\right]$ and $[\mathrm{Nd}(4-$ ab) $\left.{ }_{3}\left(\mathrm{~N}_{2} \mathrm{H}_{4}\right)\right] \cdot \mathrm{H}_{2} \mathrm{O}$ are shown in Figures $7,8,9$, and 10. From the images, it is understood that the residues are microsized metal oxides with irregular shapes.

\section{Conclusion}

The isomeric acetoxybenzoic acids and hydrazine hydrate yield the complexes of formulae, $\left[\mathrm{Ln}(3-\mathrm{ab})_{3}\left(\mathrm{~N}_{2} \mathrm{H}_{4}\right)_{2}\right] \cdot x \mathrm{H}_{2} \mathrm{O}$ where $\mathrm{Ln}=\mathrm{La}, \mathrm{Ce}, \mathrm{Pr}$ and $\mathrm{Gd}$ and $x=0 ; \mathrm{Ln}=\mathrm{Nd}$ and $\mathrm{Sm}$ and $x=1$ and $\left[\mathrm{Ln}(4-\mathrm{ab})_{3}\left(\mathrm{~N}_{2} \mathrm{H}_{4}\right)\right] \cdot \mathrm{H}_{2} \mathrm{O}$ where $\mathrm{Ln}=$ $\mathrm{La}, \mathrm{Ce}, \mathrm{Pr}, \mathrm{Nd}, \mathrm{Sm}$ and $\mathrm{Gd}$ resulting from their reaction with the respective metal nitrates. The compositions of the complexes were confirmed by elemental data. The presence of hydrazine in bidental bridging mode is inferred from their $\mathrm{N}-\mathrm{N}$ stretching frequencies. The complexes of $3-\mathrm{abH}$ release hydrazine exothermally in the temperature range $166-178^{\circ} \mathrm{C}$ whereas $4-\mathrm{abH}$ complexes undergo exothermic dehydrazination at higher temperature range $180-197^{\circ} \mathrm{C}$ indicating that the two molecules of hydrazine are tightly held between the metal atoms in four directions. All the complexes of 3 -abH undergo oxidative decomposition in the range of $437-477^{\circ} \mathrm{C}$ excepting for lanthanum, and the complexes of $4-\mathrm{abH}$ in the range of $490-550^{\circ} \mathrm{C}$. The lowertemperature decomposition of 3 -abH complexes may be due to fuelling effect of two hydrazine molecules. The magnetic and electronic data indicate the presence of metal in the complexes. A comparable XRD data of complexes imply that they have similar type of structures. However, single crystal XRD can only confirm their structures. In our case since the complexes are insoluble in any solvent due to their polymeric nature, single crystals could not be prepared. SEMEDX analysis indicated the oxides formation in microsize.

\section{Acknowledgment}

The authors wish to acknowledge the All India Council for Technical Education (AICTE) for sponsoring this work (Grant in aid no./8023/BOR/RID/RPS-2, 2008-2009).

\section{References}

[1] J. R. J. Sorenson, "Copper chelates as possible active forms of the antiarthritic agents," Journal of Medicinal Chemistry, vol. 19, no. 1, pp. 135-148, 1976.

[2] D. A. Williams, D. T. Walz, and W. O. Foye, "Synthesis and biological evaluation of tetrakis(acetylsalicylato) $\mu$ dicopper(II)," Journal of Pharmaceutical Sciences, vol. 65, no. 1, pp. 126-129, 1976.

[3] K. D. Rainsford and M. W. Whitehouse, "Concerning the merits of copper aspirin as a potential anti inflammaotry drug," Journal of Pharmacy and Pharmacology, vol. 28, no. 1, pp. 83-86, 1976.

[4] G. Smith, D. P. Arnold, C. H. L. Kennard, and T. C. W. Mak, "Tin(IV) porphyrin complexes-IV. Crystal structures of meso-tetraphenylporphyrinatotin(IV) complexes with hydroxide, water, benzoate, salicylate and acetylsalicylate as axial ligands," Polyhedron, vol. 10, no. 4-5, pp. 509-516, 1991.

[5] N. Arunadevi and S. Vairam, "3-hydroxy-2-naphthoate complexes of transition metals with hydrazine-preparation, spectroscopic and thermal studies," E-Journal of Chemistry, vol. 6, supplement 1, pp. S413-S421, 2009.

[6] S. Vairam, T. Premkumar, and S. Govindarajan, “Trimellitate complexes of divalent transition metals with hydrazinium cation thermal and spectroscopic studies," Journal of Thermal Analysis and Calorimetry, vol. 100, no. 3, pp. 955-960, 2010.

[7] S. Vairam, T. Premkumar, and S. Govindarajan, "Preparation and thermal behaviour of divalent transition metal complexes of pyromellitic acid with hydrazine," Journal of Thermal Analysis and Calorimetry, vol. 101, no. 3, pp. 979-985, 2010. 
[8] B. N. Sivasankar and S. Govindarajan, "Acetate and malonate complexes of cobalt(II), nickel(II) and zinc(II) with hydrazinium cation: preparation, spectral and thermal studies," Journal of Thermal Analysis, vol. 48, no. 6, pp. 1401-1413, 1997.

[9] S. Yasodhai and S. Govindarajan, "Coordination compounds of some divalent metals with hydrazine and dicarboxylate bridges," Synthesis and Reactivity in Inorganic and MetalOrganic Chemistry, vol. 30, no. 4, pp. 745-760, 2000.

[10] K. Kuppusamy and S. Govindarajan, "Benzoate complexes of dipositive first row transition metal ions with hydrazine," Synthesis and Reactivity in Inorganic and Metal-Organic Chemistry, vol. 26, no. 2, pp. 225-243, 1996.

[11] K. Kuppusamy and S. Govindarajan, "Hydrazinium cation as a ligand preparation and spectral, thermal and XRD studies on hydrazinium metal phthalates," Europe Journal of Solid State and Inorganic Chemistry, vol. 32, pp. 997-1012, 1995.

[12] I. A. Vogel, A Text Book of Quantitative Inorganic Analysis, Longmans, London, UK, 1975.

[13] W. T. Carnall, P. R. Fields, and K. Rajnak, "Spectral intensities of the trivalent lanthanides and actinides in solution. II. $\mathrm{Pm}^{3+}$, $\mathrm{Sm}^{3+}, \mathrm{Eu}^{3+}, \mathrm{Gd}^{3+}, \mathrm{Tb}^{3+}, \mathrm{Dy}^{3+}$, and $\mathrm{Ho}^{3+}$, The Journal of Chemical Physics, vol. 49, no. 10, pp. 4400-4403, 1968.

[14] S. P. Tanton and P. C. Mehta, "Study of some $\mathrm{Nd}^{3+}$ complexes : interelectronic repulsion, spin orbit interaction, bonding, and electronic energy levels," Journal Chemical Physics, vol. 52, pp. 4896-4902, 1968.

[15] J. E. Huheey, E. A. Keiter, R. L. Keiter, O. K. Medhi et al., Inorganic Chemistry-Principles of Structure and Reactivity, vol. 488, Dorling Kindersley, New Delhi, India, 2007.

[16] J. L. Meier, C. E. Coughenour, J. A. Carlisle, and G. O. Carlisle, "The magnetic properties of a series of copper(II) aspirinates," Inorganica Chimica Acta, vol. 106, no. 3, pp. 159-163, 1985.

[17] A. L. Abuhijleh, C. Woods, E. Bogas, and G. Le Guenniou, "Synthesis, characterization and catecholase-mimetic activity of mononuclear copper(II) aspirinate complexes," Inorganica Chimica Acta, vol. 195, no. 1, pp. 67-71, 1992.

[18] S. H. Tarulli, O. V. Quinzani, J. Dristas, and E. J. Baran, “Thermal behaviour of copper(II) complexes of haloaspirinates," Journal of Thermal Analysis and Calorimetry, vol. 60, no. 2, pp. 505-515, 2000.

[19] B. Raju and B. N. Sivasankar, "Spectral, thermal and X-ray studies on some new Bis-hydrazine lanthanide(III) glyoxylates," Journal of Thermal Analysis and Calorimetry, vol. 94, no. 1, pp. 289-296, 2008.

[20] A. Braibanti, F. Dallavalle, M. A. Pellinghelli, and E. Leporati, "The nitrogen-nitrogen stretching band in hydrazine derivatives and complexes," Inorganic Chemistry, vol. 7, no. 7, pp. 1430-1433, 1968.

[21] K. C. Patil, G. V. Chandrashekhar, and C. N. R. Rao, "Infrared spectra and thermal decompositions of metal acetates and dicarboxylates," Canadian Journal Chemistry, vol. 46, pp. 257-265, 1968. 

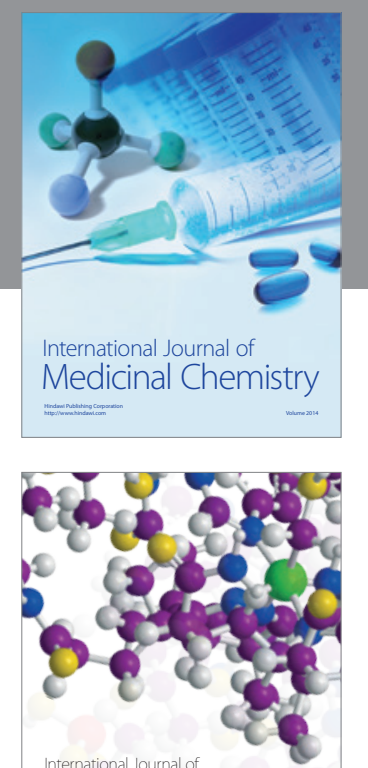

\section{Carbohydrate} Chemistry

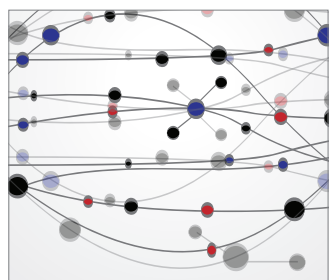

The Scientific World Journal
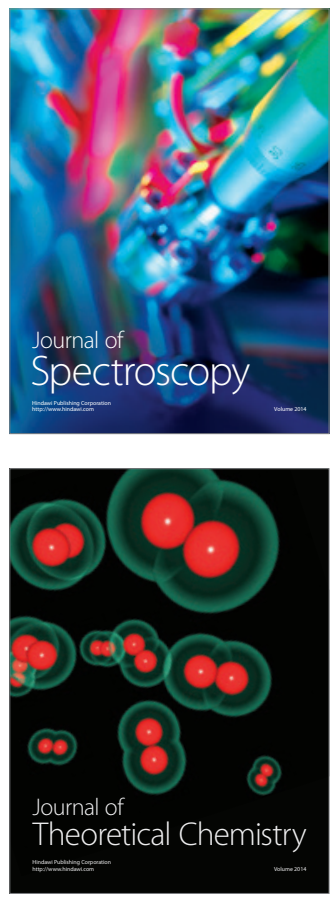
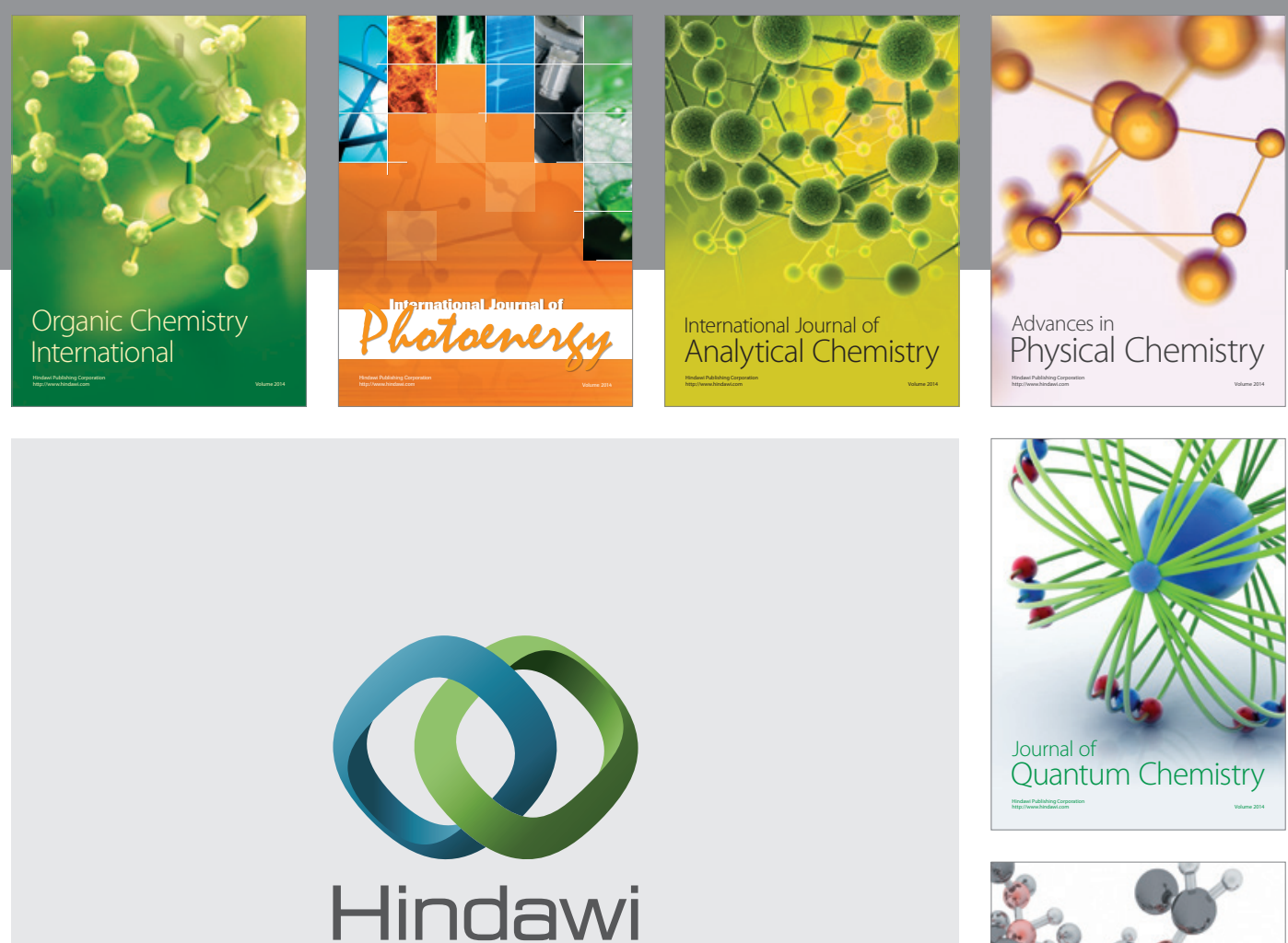

Submit your manuscripts at

http://www.hindawi.com

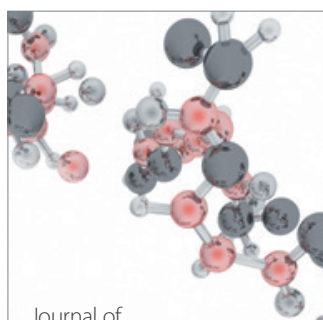

Analytical Methods

in Chemistry

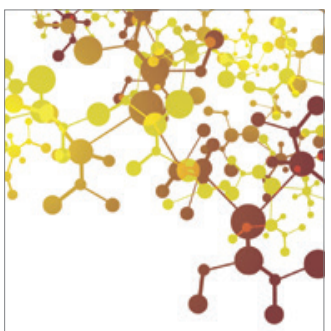

Journal of

Applied Chemistry

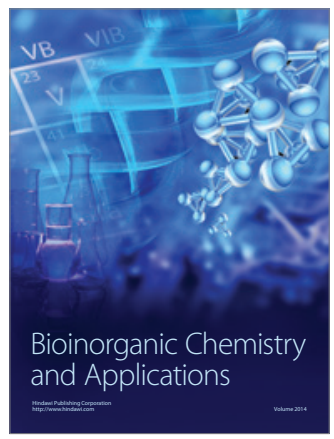

Inorganic Chemistry
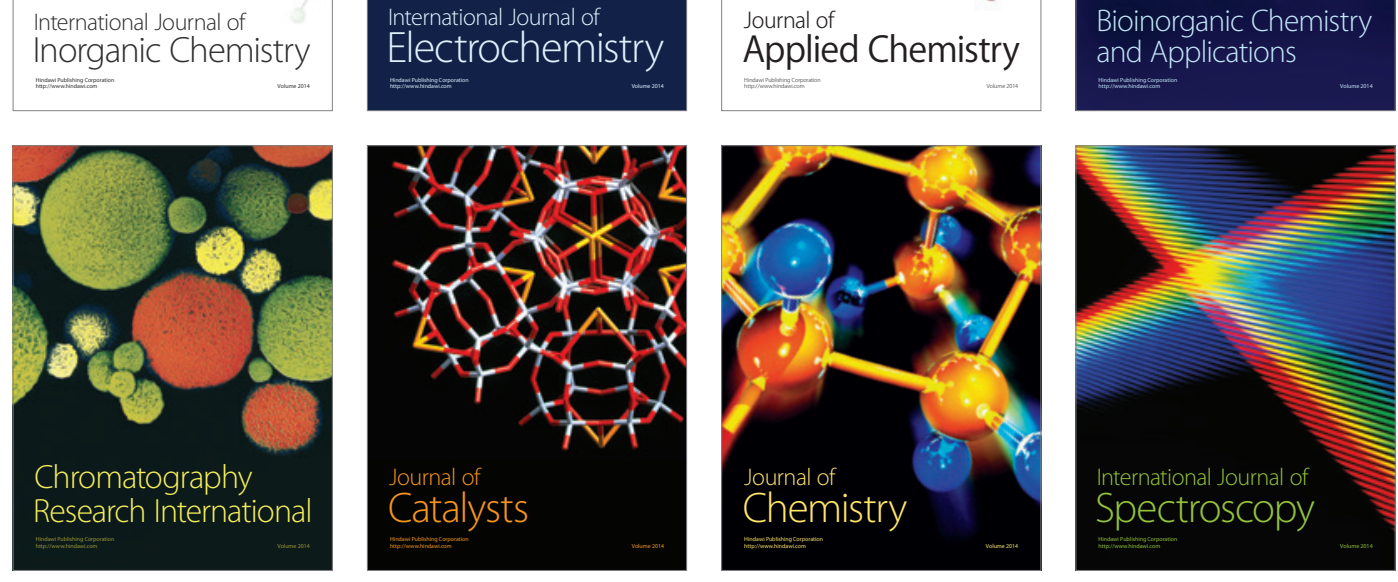